\title{
On classical de Sitter solutions in higher dimensions
}

\author{
Thomas Van Riet \\ Institut de Physique Théorique, \\ CEA Saclay, CNRS URA 2306 \\ F-91191 Gif-sur-Yvette, France \\ thomas.van-riet@cea.fr
}

\begin{abstract}
We derive necessary criteria for the existence of classical meta-stable de Sitter solutions in flux compactifications of type II supergravity down to dimensions higher than four. We find that the possibilities in higher dimensions are much more restricted than in four dimensions. The only models that satisfy the criteria are derived from $\mathrm{O} 6$ compactifications to $D=5,6$ and $\mathrm{O} 5$ compactifications to $D=5$ and no meta-stable solutions can exist in dimensions higher than six. All these models have in common that the compact dimensions are negatively curved.
\end{abstract}




\section{Contents}

1 Introduction 2

1.1 An anti-de Sitter conspiracy theory? . . . . . . . . . . . . . 2

1.2 Classical dS solutions in $D=4$ ? . . . . . . . . . . . . . 3

1.3 Classical dS solutions in $D>4$ ? . . . . . . . . . . . . . . 4

2 Tree-level energy 5

2.1 The energy sources . . . . . . . . . . . . . . . . . 5

2.2 Coupling and volume dependence of the energy . . . . . . . . . . 6

3 Existence and stability criteria $\quad 7$

3.1 Trivial example : $D=9$ with $\mathrm{D} 8 / \mathrm{O} 8$ sources . . . . . . . . . 8

3.2 Less trivial example: $D=7$ with D6/O6 sources . . . . . . . . . . . . . 9

3.3 Summary of all possibilities . . . . . . . . . . . . . . . . 10

4 dS building grounds 11

4.1 dS model I: $O 6$ planes in $D=6 \ldots \ldots \ldots \ldots \ldots \ldots$

4.2 dS model II: $O 5$ planes in $D=5 \ldots \ldots \ldots \ldots \ldots \ldots$

4.3 dS model III: $O 6$ planes in $D=5 \ldots \ldots \ldots \ldots \ldots$

5 Discussion 13

5.1 Obtained results . . . . . . . . . . . . . . . . . . . 13

5.2 Interesting problems for future research . . . . . . . . . . . . . . 14

\section{Introduction}

\subsection{An anti-de Sitter conspiracy theory?}

By now there is little doubt that string theory has an enormous (possibly infinite) amount of meta-stable solutions with four large and six small dimensions. What is less obvious is whether any of these solutions can describe our observed universe. A popular idea is that the high amount of solutions implies that many are close (and one is equal) to our observed world since values for various observable quantities are distributed around the landscape with some non-zero probability. This vision entails a possible danger as it ignores any subtle and non-obvious property of the landscape, if any.

An important observable property of a solution is the size of the cosmological constant. All explicit and fully trustworthy solutions that have ever been constructed in string theory have a non-positive cosmological constant. One reason for this is that de Sitter solutions necessarily break all susy and are therefore more "dirty". However, non-susy and trustworthy vacua have been known for some time now (see e.g. the non-susy no-scale vacua of [1]), so the lack of susy cannot be the only explanation. Maybe the true reason is that string theory is simply very constraining when it comes to the existence of meta-stable 
de Sitter solutions. The current state of affairs is that all de Sitter proposals necessarily invoke quantum corrections that cannot be computed exactly, or feature localised sources whose backreaction is potentially problematic (see e.g. 22 11]). From this viewpoint one can understand that all proposals for de Sitter solutions that are claimed to be successful (which started with [12]) have a lack of explicitness, since the problems hide in those places where explicit computations are difficult. To fully appreciate this fact it is noteworthy that $\mathrm{dS}$ solutions, that are not even phenomenologically interesting, have not been constructed in a trustworthy and explicit way. Examples of this would be de Sitter solutions in dimensions different from four or de Sitter solutions without fine-tuned size for the cosmological constant, all of which would be very interesting play grounds for studying de Sitter space in string theory nonetheless. Hence, there seems room to believe in some "anti-de Sitter conspiracy", by which we mean that the landscape of flux vacua does not contain any meta-stable dS solution at all or, in a weaker version, that the amount of dS vacua is dramatically less than assumed sofar.

Disproving the strongest version of this conspiracy theory requires only one explicit counter example that does not have to obey any special property but being meta-stable, de Sitter and trustworthy. It is allowed to be of any dimension, to have any susy-breaking scale, or have only planets populated by purple aliens.

\subsection{Classical dS solutions in $D=4$ ?}

Perhaps the easiest place to find simple and trustworthy de Sitter solutions is at the classical level governed by the 10-dimensional supergravity approximation. In this setup, the Maldacena-Nunez nogo-theorem [13] (see also [14]) gives us a useful lead: a solution with a time-independent, regular, compact space without a boundary should involve branesources.

The idea that four-dimensional Sitter solutions could arise at the purely classical level was revived in [15]. Shortly after this, some attempts were made to find explicit solutions from orientifolds of negatively curved extra dimensions [16, 17]. However these solutions can be shown to explicitly fail to solve the 10-dimensional equations of motion. A safer approach is to use either directly the 10-dimensional equations of motion with smeared sources, or, equivalently, use a consistently truncated effective action in the lower dimension. Few de Sitter solutions have been constructed within the first [18, 19] and second approach [20 22] (see [23, 24] for more related work). A review and extension of these solutions has been presented in [25]1. Those solutions that were obtained with purely geometrical ingredients (fluxes, branes and curved spaces) and for which a consistent truncation exists that allows a trustworthy computation of moduli masses, have all been shown to be perturbatively unstable. The known nogo theorems against stability [29 32] are evaded in the quoted examples and we therefore still lack a simple explanation for the presence of tachyons. On the other hand, whenever supersymmetry is broken there is no particular reason for a solution to be meta-stable. Even more, if one makes the naive

\footnotetext{
${ }^{1}$ See also references [26] 28 for examples that break susy at the compactification scale.
} 
assumption that the signs of moduli masses are randomly distributed then meta-stable solutions will be rare since four-dimensional models generically have many modul: 2 .

Even more, if a meta-stable solution will be found in the future, the approximation of smeared orientifolds, might be invalid [4, 5, ,7, 11. In any case, at the moment there is no trustworthy, meta-stable, classical de Sitter solution known 3 . For now we ignore the issues of smeared orientifolds and backreaction of localised sources and we will be satisfied with classical de Sitter solutions with smeared sources that are perturbatively stable.

\subsection{Classical dS solutions in $D>4$ ?}

Our interest for flux vacua in dimensions higher than four is mainly coming from the increase of simplicity when one goes higher in dimension. This simplicity arises because there are much less moduli and the possibilities to wrap branes and fluxes in the compact dimensions is less. Because this paper will strictly be concerned with flux compactifications down to dimensions higher than four we list the various motivations for this:

- As explained above, to disprove a hypothetical anti-de Sitter conspiracy in string theory, it suffices to find one de Sitter solution, which should be easier in dimensions higher than four since there are less moduli.

- A simple classical de Sitter solution in string theory will be very useful as an example to study the hypothetical dS/CFT correspondence [42]. For this purpose the dimension of the de Sitter solution does not have to be four.

- Meta-stable de Sitter solutions in dimensions higher than four can be dimensionally reduced to meta-stable quintessence solutions in four dimensions [43, 44]. Or in some cases meta-stable de Sitter solutions in five dimensions can be linked to meta-stable de Sitter solutions in four dimensions [45].

- It has been shown that under certain circumstances the decay of higher-dimensional de Sitter solutions can give a dynamical mechanism for compactification [46].

The rest of this paper is organised as follows. In the next section we review some basic properties of orientifold compactifications that will be needed. In section 3 we derive the necessary existence and meta-stability criteria for de Sitter solutions in dimensions higher than four. In section 4 we discuss in some details the models that fulfill our necessary criteria. We end with section 5 where we discuss the obtained results and the interesting directions for further research they imply.

\footnotetext{
${ }^{2}$ It is useful to consider the situation for extended gauged supergravities. The only models that have meta-stable de Sitter solutions occur for $\mathcal{N} \preceq 2$ supergravity 33 35. (see also 36]). Unfortunately the higher-dimensional origin of these meta-stable de Sitter solutions is not known.

${ }^{3}$ There are some recent claims of simple classical warped de Sitter solutions [37,38. If these examples originate from higher dimensional supergravity they are either necessarily singular solutions or they are to be regarded as non-compactifications, as in 39. It has been explicitly shown that the de Sitter solutions of 37] are of the form of a curved brane with de Sitter worldvolume [40. This could still be of relevance for a brane-world type scenario [1].
} 


\section{Tree-level energy}

\subsection{The energy sources}

At the classical level there are three ingredients that enter the energy of the lower-dimensional theory: fluxes, branes and curvature of the extra dimensions. Experience with compactifications down to four-dimensions have demonstrated that typical ingredients required for de Sitter solutions are negative tension objects, like orientifold planes, and negatively curved compact dimensions [16,17]. Below we will find similar results for higher dimensions. Since orientifolds are crucial we briefly repeat the most essential properties that we need.

An obvious restriction on the possible $O p$ planes for compactifications down to $D$ dimensions is that $p>D-2$, otherwise the source can not fill the $D$-dimensional space. When it comes to models with orientifold solutions of different types one can use the intersection rules for branes in flat space to understand what kind of mixtures are possible 4 . The following combined sources could be possible

$$
\begin{aligned}
& D=5: 04 / 08,06 / 08,05 / 09 \\
& D=6: 05 / 09,06 / 08
\end{aligned}
$$

and no mixture of $O p$-plane types in higher dimensions. In what follows we discard all models with $O 9$ planes since the $\mathrm{O} 9$ tadpole can only be canceled by introducing sixteen D9 branes that cancel the negative tension.

The standard way to cancel the tadpole of $\mathrm{O} p$ planes with $p<7$, without having to cancel the negative tension as well, uses fluxes. The Bianchi identity for the RR $(8-p)$-form field strength, that is magnetically sourced by an $O p$-plane, is given by

$$
\mathrm{d} F_{8-p}=H \wedge F_{6-p}+J_{O p}
$$

where $J_{O p}$ symbolizes the orientifold brane source and corresponds to a singular (deltafunction-like) form or to a regular form in the smeared approximation. The tadpole condition arises when the Bianchi identity is integrated over the compact cycle perpendicular to the source. Clearly the integrated flux combination $H \wedge F_{6-p}$ can cancel the charge (integrated source form) for suitable fluxes. However, this is not possible for $O 7-$ and O8-planes.5. The only way to solve the tadpole condition (without loosing the negative tension) in these cases, would be by considering pairs of $O p$ and anti- $O p$ planes or to make the $O p$ planes wrap cycles for which the source form $J_{O p}$ is exact 6 .

Finally we recall the specific transformation properties of the fluxes under the $O p$ targetspace involutions. The position of the $O p$-plane is given by the surface that is invariant under the target space involution $\sigma$. For all $O p$ planes the $H$-flux must be odd

\footnotetext{
${ }^{4}$ We assume that violating these restrictions make the orientifold wrap cycles that are not calibrated, which probably indicates an inconsistency or at least an instability of the model.

${ }^{5}$ The same problem occurs for NS5 branes.

${ }^{6}$ In the case of nilmanifolds there are Op solutions known that wrap a 1-cycle whose volume form is not closed and whose Hodge-dual form $J_{O p}$ then becomes exact, see e.g. [5] .
} 
$\sigma(H)=-H$. To discuss the parity of the RR form field strengths $F_{i}$ we follow [47] and introduce the operator $\alpha$ that reverses the indices of a form. Explicitly this means that

$$
\begin{array}{ll}
\alpha\left(F_{i}\right)=+F_{i}, & i=0,1,4,5,8,9, \\
\alpha\left(F_{i}\right)=-F_{i}, & i=2,3,6,7 .
\end{array}
$$

We then have the following parity conditions

$$
\begin{aligned}
& \sigma\left(F_{i}\right)=+\alpha\left(F_{i}\right), \quad(O 2,03,06, O 7), \\
& \sigma\left(F_{i}\right)=-\alpha\left(F_{i}\right), \quad(O 0, O 1, O 4, O 5, O 8, O 9) \text {. }
\end{aligned}
$$

Given that the source form $J_{p}$ is even for odd $p$ and odd for even $p$ we find that the parity rules are nicely consistent with the Bianchi-identity, which ensures that we can always employ fluxes to cancel the charge tadpole7.

\subsection{Coupling and volume dependence of the energy}

Our starting point is the 10-dimensional supergravity action of type II string theory in string frame

$$
S=\int \exp (-2 \phi) \sqrt{-g}\left(R+(4 \partial \phi)^{2}-\frac{1}{2} \frac{1}{3 !} H^{2}\right)-\int \sqrt{-g} \frac{1}{2} \sum_{q} \frac{1}{q !}\left|F_{q}^{R R}\right|^{2},
$$

where $q$ runs over $0,2,4$ for type IIA sugra and over 1,3,5 for type IIB supergravity, with the usual caveat for the self-dual 5-form field strength. We suppressed the Chern-Simons terms as we will not need them. The string coupling constant is given by $g_{s}=\exp (\phi)$. There are also localised sources whose action can be added to the bulk action. The only piece we need is the DBI part that couples to the metric. For $O p$ and $D p$ sources this is given by

$$
S_{D p / O p}=-T_{p} \int_{p} \exp (-\phi) \sqrt{g_{p+1}}
$$

where $g_{p+1}$ is the induced metric on the source worldvolume and $T_{p}$ is the tension, which is negative for $O p$ sources and positive for $D p$ sources. We can also consider NS5 branes, whose action differs from the $D 5$ brane by an extra power of $\exp (-\phi)$.

In the unwarped limit, the 10-dimensional metric, describing a compactification downto $D$ dimensions, can be written as follows

$$
\mathrm{d} s_{10}^{2}=\tau^{-2} \mathrm{~d} s_{D}^{2}+\rho \mathrm{d} s_{10-D}^{2} .
$$

The modulus $\rho$ is the string frame volume and consequently we normalised $\int \sqrt{g_{10-D}}$ to one in string units. The modulus $\tau$ can be shown to be

$$
\tau^{D-2}=\exp (-2 \phi) \rho^{\frac{10-D}{2}},
$$

\footnotetext{
${ }^{7}$ This corrects a wrong statement about $O 4$ models in 18 .
} 
in order to have $D$-dimensional Einstein frame. The variables $\rho$ and $\tau$ span a flat 2dimensional subspace of the general modulispace. In this parametrisation $\rho$ and $\tau$ do not have standard kinetic terms, but this is not required for the analysis done in this paper. We have chosen this specific parametrisation in order to make contact with the previous literature on the topic.

Using the above we can derive the dependency of the various energy contributions on the string coupling and volume modulus. The total energy $V$ is the sum of various energies $V=\sum_{i} V_{i}$. These separate energies $V_{i}$ come from the curved extra dimensions, $V_{i}=V_{R}$, the $H$-flux, $V_{i}=V_{H}$, the RR q-form fluxes $V_{i}=V_{R R}^{q}$, and the sources $V_{i}=V_{D p / O p}$ and $V_{i}=V_{N S 5}$. Specifically we find

$$
\begin{aligned}
& V_{R} \sim-\tilde{R}_{10-D}(\varphi) \rho^{-1} \tau^{-2}, \\
& V_{H} \sim|\tilde{H}|^{2}(\varphi) \rho^{-3} \tau^{-2}, \\
& V_{R R}^{q} \sim\left|\tilde{F}_{q}\right|^{2}(\varphi) \rho^{\frac{10-D}{2}-q} \tau^{-D}, \\
& V_{D p / O p} \sim T_{p}(\varphi) \rho^{\frac{2 p-D-8}{4}} \tau^{-\frac{D+2}{2}}, \\
& V_{N S 5} \sim T(\varphi) \rho^{-2} \tau^{-2} .
\end{aligned}
$$

The notation is such that the tilde contractions $|\tilde{F}|^{2}$ are done using the internal metric with the volume modulus $\rho$ factored out. Furthermore we introduced the democratic notation for fluxes that are space-filling. As an example, an $F_{4}$ flux filling four noncompact dimensions (when $D=4$ ) will be considered through its Hodge dual $F_{6}$. We have symbolically introduced the hidden dependence on the non-universal moduli as $\varphi$. These non-universal moduli could be shape moduli of the internal dimensions, or gauge potential moduli. The way they appear in the flux contribution and source contribution depends on the details of the cycles thread and wrapped by fluxes and sources.

In this paper we do not consider any KK monopoles or fractional Wilson lines as in [16. The backreaction of KK monopoles is worry some, as well is it unclear how to find the stable cycles for such branes. In our approach we stick to brane setups that lead to lower-dimensional supergravity theories. This requires the sources to be calibrated. The benefit of this restriction is that supersymmetry of the lower-dimensional action restricts the possible corrections, and secondly, that the branes are wrapped in a consistent manner.

\section{Existence and stability criteria}

With the $\rho, \tau$ dependence at hand one can deduce necessary (but not sufficient) criteria for the existence of de Sitter critical point to the potential. This boils down to analysing the constraints coming from the following two equations and one inequality

$$
\partial_{\rho} V=0, \quad \partial_{\tau} V=0, \quad V>0 .
$$

For the case $D=4$ this has been initiated in [15] and systematically worked out in [18,48]. The equations (3.1) can easily shown to coincide with specific linear combinations of the 
10-dimensional dilaton equation and the trace of the 10-dimensional Einstein equation over the compact dimensions [18].

The universal moduli $\rho$ and $\tau$ do not only allow us to find existence criteria for de Sitter solutions, they also allow us to find minimal requirements for meta-stability of the de Sitter solutions [32]. This goes as follows: if the two by two Hessian

$$
\left(\begin{array}{cc}
\partial_{\rho}^{2} V & \partial_{\rho} \partial_{\tau} V \\
\partial_{\rho} \partial_{\tau} V & \partial_{\tau}^{2} V
\end{array}\right)
$$

has a negative eigenvalue it implies that the full mass matrix will also have negative eigenvalues, through Silvesters criterium. The eigenvalues of the above two by two Hessian will not be part of the spectrum of the full $N$ by $N$ mass matrix due to moduli mixing, but this is not of any importance when it comes to finding necessary conditions for stability. In what follows it is useful to remind that the eigenvalues $\lambda_{+}, \lambda_{-}$of a symmetric two by two matrix

$$
\left(\begin{array}{cc}
t_{1} & s \\
s & t_{2}
\end{array}\right)
$$

are given by

$$
2 \lambda_{ \pm}=t_{1}+t_{2} \pm \sqrt{\left(t_{1}+t_{2}\right)^{2}-4\left(t_{1} t_{2}-s^{2}\right)}
$$

Furthermore if either $t_{1}$ or $t_{2}$ is negative so will be at least one of the eigenvalues due to Sylvester's criterium. The smallest eigenvalue is always $\lambda_{-}$and stability therefore requires

$$
\lambda_{-}>0 \quad \Leftrightarrow \quad t_{1} t_{2}>s^{2},
$$

with both $t_{1}$ and $t_{2}$ positive. If this condition is violated we cannot have a meta-stable de Sitter solution. Equation (3.5) is easy to interpret. It simply states that a negative determinant implies negative eigenvalues since the determinant equals the product of eigenvalues.

We will now list all existence and stability criteria for de Sitter solutions that can be obtained from the universal moduli. We simplify the calculations using a rescaling of the moduli. Consider a critical point of the potential at the values $\rho_{c}, \tau_{c}$ then we redefine the variables $\rho$ and $\tau$ as follow 8

$$
\rho \rightarrow \frac{\rho}{\rho_{c}}, \quad \tau \rightarrow \frac{\tau}{\tau_{c}}
$$

In this notation the critical point is always at the values $\rho=1, \tau=1$. As a consequence the coefficients in the potential are not anymore $|H|^{2},\left|F_{p}\right|^{2}$ or $T$, but are rescaled by some powers of $\rho_{c}$ and $\tau_{c}$.

\subsection{Trivial example : $D=9$ with $\mathrm{D} 8 / \mathrm{O} 8$ sources}

Consider compactifications with only one compact direction. This restricts the possible brane sources to be $D 8 / O 8$ and we are necessarily in IIA. Then the possible flux is $F_{0}$ flux.

\footnotetext{
${ }^{8}$ I am grateful to Ulf Danielsson for pointing out this simplifying trick.
} 
We write the potential as follows

$$
V=f_{0}^{2} \tau^{-9} \rho^{1 / 2}+T \tau^{-11 / 2} \rho^{-1 / 4}
$$

where, up to numerical factors and rescalings with $\rho_{c}$ and $\tau_{c}, f_{0}$ corresponds to the Romans mass and $T$ to the tension. Then we immediately find

$$
\begin{aligned}
& \partial_{\rho} V=0 \Rightarrow f_{0}^{2}=\frac{1}{2} T, \\
& \partial_{\tau} V=0 \Rightarrow f_{0}^{2}=-\frac{11}{18} T .
\end{aligned}
$$

Hence no solution is possible at all, whether dS, AdS or Minkowski. We have even been too mild here since, strictly speaking, the $F_{0}$ flux should be projected out by the $O 8$ plane and we furthermore have no way to cancel the $O 8$ tadpole without canceling the $O 8$ tension. In what follows we are more careful in taking into account the orientifold involutions and tadpole conditions.

\subsection{Less trivial example: $D=7$ with $\mathrm{D} 6 / \mathrm{O} 6$ sources}

Things start to get more interesting in $D=7$ where one possibility is to have space-filling $D 6 / O 6$ sources. The possible fluxes are $F_{0}, F_{2}$ and $H$. Note that $F_{2}$ is odd and given that it has two legs outside the $O 6$-plane it will normally not survive the orientifold projection. We nonetheless keep it with us, in case there is no $O 6$ plane or it can somehow survive 9

We write the scalar potential as

$$
V=f_{0}^{2} \tau^{-7} \rho^{3 / 2}+f_{2}^{2} \tau^{-7} \rho^{1 / 2}+h^{2} \tau^{-2} \rho^{-3}-R \tau^{-2} \rho^{-1}+T \tau^{-9 / 2} \rho^{-3 / 4} .
$$

We introduced a few new symbols: $R$ equals the curvature of the internal dimensions (up to a positive constant), $h^{2}$ equals the $H^{2}$ (again with some positive proportionality constant which we will not mention anymore) and $f_{2}^{2}$ equals $F_{2}^{2}$. We then find

$$
\begin{aligned}
& \partial_{\rho} V=0 \Rightarrow R=-\frac{3}{2} f_{0}^{2}+\frac{1}{2} f_{2}^{2}+3 h^{2}+\frac{3}{4} T, \\
& \partial_{\tau} V=0 \Rightarrow T=-\frac{10}{3} f_{0}^{2}-2 f_{2}^{2}+\frac{4}{3} h^{2} .
\end{aligned}
$$

If we plug this into the on-shell value for $V$ we find

$$
V=\frac{5}{3}\left(f_{0}^{2}-h^{2}\right)
$$

This can have any sign, so this model could allow Minkowski, AdS and dS solutions.

The Hessian is given by

$$
\partial_{i} \partial_{j} V=\left(\begin{array}{cc}
\frac{35}{8} f_{0}^{2}+\frac{1}{8} f_{2}^{2}+\frac{23}{4} h^{2} & -\frac{55}{4} f_{0}^{2}-\frac{5}{4} f_{2}^{2}+\frac{5}{2} h^{2} \\
-\frac{55}{4} f_{0}^{2}-\frac{5}{4} f_{2}^{2}+\frac{5}{2} h^{2} & -\frac{5}{2} f_{0}^{2}+\frac{25}{2} f_{2}^{2}+15 h^{2}
\end{array}\right) .
$$

\footnotetext{
${ }^{9}$ Note that localised $D 6 / O 6$ sources lead to non-trivial $F_{2}$ profiles, but this is not really counted as flux, it is rather a consequence of backreaction.
} 
When $V=0$ (and $f_{2}^{2}=0$ ) we find that $R=0$ and that the Hessian has one positive and zero eigenvalue, consistent with the fact that the scalar potential can then be written as a square

$$
V=f_{0}^{2}\left(\tau^{-7 / 2} \rho^{3 / 4}-\tau^{-1} \rho^{-3 / 2}\right)^{2}
$$

These solutions are the no-scale Minkowski solutions constructed in [5] and they exist in dimensions $D=2 \ldots 7$ and were first studied in $D=4$ [1].

In order to have de Sitter solutions we need $f_{0}^{2}>h^{2}$ and in that case we can demonstrate that the tension is necessarily negative, which corresponds to having net $O 6$ sources. Let us therefore take $f_{2}^{2}=0$ in what follows. The determinant of the Hessian is given by

$$
\operatorname{det}\left(\partial_{i} \partial_{j} V\right)=40 f_{0}^{4}\left(-5+2 \frac{h^{4}}{f_{0}^{4}}+3 \frac{h^{2}}{f_{0}^{2}}\right)
$$

De Sitter requires $h^{2} / f_{0}^{2}<1$ with the no-scale Minkowski solution at the turning point $h^{2} / f_{0}^{2}=1$. Hence we find an elegant structure: exactly at the Minkowski turning point, a tachyon appears and the de Sitter critical points can never be a local minima of the potential. These unstable de Sitter solutions could possible be engineered with pairs of $O 6$ and anti-O6 planes as pointed out in [7].

\subsection{Summary of all possibilities}

As shown in the two examples the technique is to use the two $\partial V=0$ equations to eliminate $R$ and $T$ in terms of the fluxes, which are strict positive. Then this is plugged into the on-shell value for $V$ to read of the sign of the cosmological constant. To determine stability we do the same for the Hessian.

Let us summarise the result of the computation:

1. $D>7$ : No dS critical points are possible.

2. $D=7$ : A dS critical point build from 06 planes and negative curvature is allowed but necessarily unstable as shown explicitly in the previous section.

3. $D=6: 05$ sources with negatively curved compact dimensions allow dS critical points, which are necessarily unstable. However $O 6$ sources can have critical points which are perturbatively stable in the $\rho, \tau$ directions if $F_{2}$ is large enough. The curvature of the internal dimensions is required to be negative.

4. $D=5$ : $O 4$ models with negatively curved compact dimensions again only allow unstable dS critical points, whereas $O 5$ and $O 6$ models can evade unstable directions at a dS critical point. Also here the curvature of the internal dimensions is negative.

Most relevant to observe here is that meta-stable solutions in $D>6$ cannot exist and that for $D=5,6$ there is only a small amount of models that potentially allow meta-stable de Sitter solutions. 
There is a recurring pattern in each dimension $D$ that allows a no-scale Minkowski solution with space-filling $O(D-1)$-planes (which are the values $D=2, \ldots, 7[5]$ ). These models all allow de Sitter critical points by negatively curving the compact dimensions of the no-scale solution and changing the ratio between net tension and charge of the orientifolds (such that there is more net negative tension than net negative charge). These solutions always have an unstable direction, which coincides with the massless direction of the no-scale Minkowski solution. In other words, the results of section 3.2 for the case of O6 planes in $D=7$ extends to the other dimensions as well.

We have furthermore checked that none of the mixed orientifold plane combinations mentioned in section 2 fulfill the criteria. This is straightforward for the $05 / 09$ combination since we discarded solutions with net $O 9$ tension as the $O 9$ tadpole cannot be canceled without canceling the $O 9$ tension. The $06 / O 8$ combinations naively fulfill the criteria in $D=5$ and $D=6$ since $O 6$ planes separately do. However the presence of the $O 8$ plane implies that the necessary $F_{0}$ flux is projected out and that tadpole constraint cannot be solved easily. The same tadpole constraint problem is there for the $04 / O 8$ model in $D=5$. Upon neglecting tadpole constraints the $O 4 / O 8$ combination does satisfy the criteria for de Sitters solutions that are stable in the $(\rho, \tau)$-directions.

\section{4 dS building grounds}

In this section we provide some details of the models in $D=5$ and $D=6$ that potentially allow meta-stable dS solutions.

\section{1 dS model I: $O 6$ planes in $D=6$}

In this case the $O 6$ planes wrap a one-cycle which projects out any $F_{4}$ flux, since the flux would have one leg along the 1-cycle wrapped by the $O 6$, giving it odd parity. The remaining fluxes are $H, F_{0}$ and $F_{2}$. The $\partial V=0$ equations lead to

$$
\begin{aligned}
& R=\frac{11}{3}\left(h^{2}-f_{0}^{2}\right)-f_{2}^{2}, \\
& 3 T=-10 f_{0}^{2}-6 f_{2}^{2}+4 h^{2} .
\end{aligned}
$$

When plugged into the potential we find

$$
V=\frac{4}{3}\left(f_{0}^{2}-h^{2}\right)
$$

which shows that dS solutions require $f_{0}^{2}>h^{2}$ and imply negative internal curvature $R<0$ and net $O 6$-plane tension $T<0$.

The necessary stability condition (3.5) requires us to compute the determinant of the Hessian. The Hessian is given by

$$
\partial_{i} \partial_{j} V=\left(\begin{array}{cc}
\frac{41}{6} f_{0}^{2}+\frac{17}{3} h^{2}+\frac{1}{2} f_{2}^{2} & -\frac{34}{3} f_{0}^{2}+\frac{4}{3} h^{2}-2 f_{2}^{2} \\
-\frac{34}{3} f_{0}^{2}+\frac{4}{3} h^{2}-2 f_{2}^{2} & -\frac{8}{3} f_{0}^{2}+\frac{32}{3} h^{2}+8 f_{2}^{2}
\end{array}\right) .
$$


For the Minkowski solutions $f_{0}^{2}=h^{2}$, the determinant of the Hessian simplifies to

$$
\operatorname{det}\left(\partial_{i} \partial_{j} V\right)=64 f_{0}^{2} f_{2}^{2}
$$

which demonstrates that the Minkowski solutions are stable in the $\rho, \tau$-directions, and that the solution is no-scale when either $f_{0}$ or $f_{2}$ vanish. For the case $f_{0}^{2}=h^{2}=0$ and $f_{2} \neq 0$ this no-scale solution has been explicitly constructed in [5] from T-dualising GKP [1] and this solution corresponds to an $O 6$ wrapping a one-cycle in a nilmanifold.

We can verify that the Hessian can be positive definite for de Sitter solutions by slightly perturbing the Minkowski solutions to:

$$
f_{0}^{2}=h^{2}+\delta, \quad \delta>0 .
$$

Where we think of $\delta$ as arbitrary tiny and positive. At first-order in $\delta$ the determinant then becomes

$$
\operatorname{det}\left(\partial_{i} \partial_{j} V\right)=64 h^{2} f_{2}^{2}-\frac{616}{3} h^{2} \delta+8 f_{2}^{2} \delta .
$$

Hence it is a simple consequence of continuity of the determinant of the Hessian that de Sitter solutions that are stable in the $(\rho, \tau)$-directions exist when perturbing the no-scale Minkowski vacua given by $f_{0}=h=0$ and $f_{2} \neq 0$. This is an interesting place to look for dS solutions since solutions could be tuned very close to a (susy) Minkowski solution.

\section{2 dS model II: $O 5$ planes in $D=5$}

This situation is as good as identical to the 06 planes in $D=6$, with the roles of the $F_{0}$ and $F_{2}$ flux now played by the $F_{1}$ and the $F_{3}$ fluxes. First one observes that the $F_{5}$-flux must be projected out (just like the $F_{4}$ before). The $\partial V=0$ equations lead to

$$
\begin{aligned}
R & =\frac{9}{2} h^{2}-\frac{9}{2} f_{1}^{2}-f_{3}^{2}, \\
T & =2 h^{2}-4 f_{1}^{2}-2 f_{3}^{2}, \\
V & =\frac{3}{2}\left(f_{1}^{2}-h^{2}\right) .
\end{aligned}
$$

Again this implies that de Sitter solutions have negatively curved internal dimensions and net orientifold tension. Minkowski solutions exist whenever $f_{1}=h$. The Hessian is given by

$$
\partial_{i} \partial_{j} V=\left(\begin{array}{cc}
\frac{45}{8} h^{2}+\frac{9}{2} f_{1}^{2}+\frac{1}{8} f_{3}^{2} & \frac{9}{4} h^{2}-9 f_{1}^{2}-\frac{3}{4} f_{3}^{2} \\
\frac{9}{4} h^{2}-9 f_{1}^{2}-\frac{3}{4} f_{3}^{2} & \frac{21}{2} h^{2}-6 f_{1}^{2}+\frac{9}{2} f_{3}^{2}
\end{array}\right) .
$$

This Hessian has the same structure as the example above. When slightly perturbing the Minkowski solutions towards de Sitter solutions by $f_{1}^{2}=h^{2}+\delta$, where $\delta>0$, the Hessian simplifies and the determinant becomes

$$
\operatorname{det}\left(\partial_{i} \partial_{j} V\right)=36 h^{2} f_{3}^{2}-162 h^{2} \delta+6 f_{3}^{2} \delta .
$$

We notice that the perturbation of the no-scale solutions with with $f_{1}=h=0$ (and $\left.f_{3} \neq 0\right)$ can give de Sitter solutions which are stable in the $(\rho, \tau)$-directions. 


\section{3 dS model III: $O 6$ planes in $D=5$}

The two $\partial V=0$ equations entail

$$
\begin{aligned}
R & =\frac{10}{3} h^{2}-\frac{10}{3} f_{0}^{2}-f_{2}^{2}+\frac{4}{3} f_{4}^{2}, \\
T & =\frac{4}{3} h^{2}-\frac{10}{3} f_{0}^{2}-2 f_{2}^{2}-\frac{2}{3} f_{4}^{2}, \\
V & =f_{0}^{2}-h^{2}-f_{4}^{2} .
\end{aligned}
$$

As before, we find that de Sitter solutions necessarily require negative curvature $(R<0)$ and negative tension $(T<0)$. The Hessian is given by

$$
\partial_{i} \partial_{j} V=\left(\begin{array}{cc}
\frac{23}{4} h^{2}+\frac{75}{8} f_{0}^{2}+\frac{9}{8} f_{2}^{2}+\frac{7}{8} f_{4}^{2} & \frac{1}{2} h^{2}-\frac{35}{4} f_{0}^{2}-\frac{9}{4} f_{2}^{2}+\frac{17}{4} f_{4}^{2} \\
\frac{1}{2} h^{2}-\frac{35}{4} f_{0}^{2}-\frac{9}{4} f_{2}^{2}+\frac{17}{4} f_{4}^{2} & 7 h^{2}-\frac{5}{2} f_{0}^{2}+\frac{9}{2} f_{2}^{2}+\frac{23}{2} f_{4}^{2}
\end{array}\right) .
$$

The Minkowski solutions are defined by

$$
f_{0}^{2}=h^{2}+f_{4}^{2}
$$

In this case the determinant of the Hessian is given by

$$
\operatorname{det}\left(\partial_{i} \partial_{j} V\right)=9\left(8 f_{4}^{4}+4 h^{2} f_{2}^{2}+12 h^{2} f_{4}^{2}+4 f_{4}^{2} f_{2}^{2}\right) .
$$

Hence dS points close by are stable in the $(\rho, \tau)$-directions. Note that no-scale Minkowski solutions are defined by $h^{2}=f_{0}^{2}=f_{4}^{2}$ and only $f_{2}^{2} \neq 0$. Perturbing those no-scale solutions into the $\mathrm{dS}$ regime by $f_{0}^{2}=\delta$ leads to an instability in the $(\rho, \tau)$-directions.

\section{Discussion}

\subsection{Obtained results}

In this paper we derived necessary (but not sufficient) conditions for the existence of classical meta-stable de Sitter solutions in $D>4$ dimensions from orientifold compactifications of type II supergravity. The necessary conditions are derived from the universal dependence of the scalar potential on the dilaton and volume modulus. This is a continuation of the results known for compactifications to $D=4$, as derived in [15, 18, 32, 48, for which we have simplified the method significantly.

The results show that no meta-stable de Sitter solution in $D>6$ can exist and that only few possibilities in $D=5,6$ can work. The three cases that fulfill the necessary criteria (O6 in $D=5,6$ and $O 5$ in $D=5$ ) have in common that the net source tension has to be negative and that the curvature of the internal dimensions has to be negative. This is in line with almost all examples in $D=410$.

\footnotetext{
${ }^{10}$ We have noticed that the $D=4$ examples were negative curvature is not required probably require fluxes that are projected out by the orientifold or require difficult-to-satisfy tadpole constraints.
} 
Some of the criteria can be weakened when wrapped NS5 branes are included. We have not explicitly listed the new possibilities that arise with NS5 branes, because NS5 branes are difficult to incorporate in simple models. This comes from the NS5 tadpole condition, which cannot be satisfied using fluxes. Therefore one necessarily has to wrap the NS5 branes on trivial cycles or allow anti-NS5 branes in homologous cycles without the branes annihilating. Such possibilities are not easy to construct in a trustworthy manner [49].

An important drawback of our derivation is the smeared (unwarped) approximation. This approximation works for deriving BPS solutions [1,5], but could easily be problematic for non-BPS solutions [7,11]. This is especially easy to spot from the 10-dimensional Einstein equations for dS solutions from orientifolds of negatively curved compact dimensions [4, which comprises all our examples.

\subsection{Interesting problems for future research}

The obtained results suggest many different directions for further research, which we list here:

- Both the $O 6$ in $D=6$ model and the $O 5$ in $D=5$ model have Minkowski vacua and the would-be de Sitter solutions close to those Minkowski vacua are stable in the coupling and volume directions. However, preliminary investigations similar to the ones in [5] indicate that dS solutions might be excluded in these models11. This would point to the very exciting possibility that only the $O 6$ model in $D=5$ is left.

- Our criteria are derived from the volume modulus and dilaton. But clearly, for every scalar field that is added one obtains a new stability and existence criterium. It should be possible to add one more "universal" scalar field, without having to fix the geometry and topology of the model. Such a scalar field could be the volume of the cycle wrapped by the orientifold.

- An obvious method that presents itself is to classify 4 and 5 dimensional group manifolds that allow the proper $O 5$ and 06 involutions, similar to the investigation in [25, 27]. This allows to scan a large set of models for de Sitter solutions. We hope to report on this in the future.

- It would be useful to compare the existence and stability issues for de Sitter solutions from type II orientifolds with those from $\alpha^{\prime}$-corrections in heterotic supergravity as these should be dual to each other 12 . In this respect the results of the recent paper [50] indicate that meta-stable de Sitter solutions can be ruled out in large regions of parameter space.

\footnotetext{
${ }^{11}$ This is similar to the way dS solutions can be excluded in IIB supergravity with BPS D3/O3 sources 1]

${ }^{12}$ I would like to thank Callum Quigley for pointing this out.
} 


\section{Acknowledgments}

I have benefited from discussions with Ulf Danielsson and Timm Wrase and I am especially grateful to Timm Wrase for spotting errors and typos in the first draft. My work is supported by the ERC Starting Independent Researcher Grant 259133-ObservableString.

\section{References}

[1] S. B. Giddings, S. Kachru and J. Polchinski, Hierarchies from fluxes in string compactifications, Phys. Rev. D66 (2002) 106006 hep-th/0105097.

[2] P. McGuirk, G. Shiu and Y. Sumitomo, Non-supersymmetric infrared perturbations to the warped deformed conifold, Nucl.Phys. B842 (2010) 383-413 [0910.4581].

[3] I. Bena, M. Grana and N. Halmagyi, On the Existence of Meta-stable Vacua in Klebanov-Strassler, JHEP 1009 (2010) 087 [0912.3519].

[4] M. R. Douglas and R. Kallosh, Compactification on negatively curved manifolds, JHEP 06 (2010) 004 [1001.4008].

[5] J. Blaback, U. H. Danielsson, D. Junghans, T. Van Riet, T. Wrase and M. Zagermann, Smeared versus localised sources in flux compactifications, JHEP 12 (2010) 043 [1009.1877].

[6] I. Bena, G. Giecold and N. Halmagyi, The Backreaction of Anti-M2 Branes on a Warped Stenzel Space, JHEP 04 (2011) 120 [1011.2195].

[7] J. Blaback, U. H. Danielsson, D. Junghans, T. Van Riet, T. Wrase and M. Zagermann, The problematic backreaction of SUSY-breaking branes, 1105.4879.

[8] G. Giecold, E. Goi and F. Orsi, Assessing a candidate IIA dual to metastable supersymmetry-breaking, 1108.1789 .

[9] S. Massai, Metastable Vacua and the Backreacted Stenzel Geometry, 1110.2513.

[10] C. P. Burgess, A. Maharana, L. van Nierop, A. A. Nizami and F. Quevedo, On Brane Back-Reaction and de Sitter Solutions in Higher-Dimensional Supergravity, 1109.0532.

[11] J. Blaback et al., (Anti-)Brane backreaction beyond perturbation theory, 1111.2605.

[12] S. Kachru, R. Kallosh, A. D. Linde and S. P. Trivedi, De Sitter vacua in string theory, Phys.Rev. D68 (2003) 046005 hep-th/0301240).

[13] J. M. Maldacena and C. Nunez, Supergravity description of field theories on curved manifolds and a no go theorem, Int. J. Mod. Phys. A16 (2001) 822-855 hep-th/0007018. 
[14] B. de Wit, D. J. Smit and N. D. Hari Dass, Residual Supersymmetry of Compactified $D=10$ Supergravity, Nucl. Phys. B283 (1987) 165.

[15] M. P. Hertzberg, S. Kachru, W. Taylor and M. Tegmark, Inflationary constraints on type IIA string theory, JHEP 12 (2007) 095 [0711.2512].

[16] E. Silverstein, Simple de Sitter Solutions, Phys. Rev. D77 (2008) 106006 [0712.1196].

[17] S. S. Haque, G. Shiu, B. Underwood and T. Van Riet, Minimal simple de Sitter solutions, Phys. Rev. D79 (2009) 086005 [0810.5328].

[18] U. H. Danielsson, S. S. Haque, G. Shiu and T. Van Riet, Towards classical de Sitter solutions in string theory, JHEP 09 (2009) 114 [0907.2041].

[19] U. H. Danielsson, P. Koerber and T. Van Riet, Universal de Sitter solutions at tree-level, JHEP 05 (2010) 090 [1003.3590].

[20] R. Flauger, S. Paban, D. Robbins and T. Wrase, Searching for slow-roll moduli inflation in massive type IIA supergravity with metric fluxes, Phys. Rev. D79 (2009) 086011 [0812.3886].

[21] C. Caviezel, P. Koerber, S. Körs, D. Lüst, T. Wrase and M. Zagermann, On the cosmology of type IIA compactifications on SU(3)-structure Manifolds, JHEP 04 (2009) 010 [0812.3551].

[22] C. Caviezel, T. Wrase and M. Zagermann, Moduli stabilization and cosmology of type IIB on SU(2)-structure orientifolds, JHEP 04 (2010) 011 [0912.3287].

[23] B. de Carlos, A. Guarino and J. M. Moreno, Complete classification of Minkowski vacua in generalised flux models, JHEP 02 (2010) 076 [0911.2876].

[24] G. Dibitetto, R. Linares and D. Roest, Flux compactifications, gauge algebras and de Sitter, Phys. Lett. B688 (2010) 96-100 [1001.3982].

[25] U. H. Danielsson, S. S. Haque, P. Koerber, G. Shiu, T. Van Riet et al., De Sitter hunting in a classical landscape, Fortsch.Phys. 59 (2011) 897-933 [1103.4858].

[26] A. Saltman and E. Silverstein, A new handle on de Sitter compactifications, JHEP 01 (2006) 139 hep-th/0411271.

[27] D. Andriot, E. Goi, R. Minasian and M. Petrini, Supersymmetry breaking branes on solvmanifolds and de Sitter vacua in string theory, 1003.3774.

[28] X. Dong, B. Horn, E. Silverstein and G. Torroba, Micromanaging de Sitter holography, Class. Quant. Grav. 27 (2010) 245020 [1005.5403]. 
[29] L. Covi et al., de Sitter vacua in no-scale supergravities and Calabi-Yau string models, JHEP 06 (2008) 057 [0804.1073].

[30] M. Gomez-Reino, J. Louis and C. A. Scrucca, No metastable de Sitter vacua in N=2 supergravity with only hypermultiplets, JHEP 02 (2009) 003 [0812.0884].

[31] A. Borghese and D. Roest, Metastable supersymmetry breaking in extended supergravity, JHEP 05 (2011) 102 [1012.3736].

[32] G. Shiu and Y. Sumitomo, Stability Constraints on Classical de Sitter Vacua, 1107.2925 .

[33] P. Fre, M. Trigiante and A. Van Proeyen, Stable de Sitter vacua from $N=2$ supergravity, Class. Quant. Grav. 19 (2002) 4167-4194 hep-th/0205119.

[34] M. Gunaydin and M. Zagermann, The Vacua of 5-D, N=2 gauged Yang-Mills/Einstein tensor supergravity: Abelian case, Phys.Rev. D62 (2000) 044028 hep-th/0002228.

[35] B. Cosemans and G. Smet, Stable de Sitter vacua in $N=2, D=5$ supergravity, Class.Quant.Grav. 22 (2005) 2359-2380 hep-th/0502202].

[36] D. Roest and J. Rosseel, De Sitter in Extended Supergravity, Phys. Lett. B685 (2010) 201-207 [0912.4440].

[37] I. P. Neupane, Warped compactification on curved manifolds, Class. Quant. Grav. 28 (2011) 125015 [1006.4495].

[38] M. Minamitsuji and K. Uzawa, Warped de Sitter compactifications and modulus stabilization, 1109.4818 .

[39] G. W. Gibbons and C. M. Hull, de Sitter space from warped supergravity solutions, hep-th/0111072.

[40] W. Chemissany, B. Janssen and T. Van Riet, Einstein Branes, JHEP 10 (2011) 002 [1107.1427].

[41] I. P. Neupane, De Sitter brane-world, localization of gravity, and the cosmological constant, Phys. Rev. D83 (2011) 086004 [1011.6357].

[42] A. Strominger, The dS/CFT correspondence, JHEP 10 (2001) 034 hep-th/0106113.

[43] P. K. Townsend, Quintessence from M-theory, JHEP 11 (2001) 042 hep-th/0110072.

[44] J. Rosseel, T. Van Riet and D. B. Westra, Scaling cosmologies of $N=8$ gauged supergravity, Class. Quant. Grav. 24 (2007) 2139-2152 hep-th/0610143. 
[45] O. Ogetbil, Stable de Sitter Vacua in 4 Dimensional Supergravity Originating from 5 Dimensions, Phys. Rev. D78 (2008) 105001 [0809.0544].

[46] S. M. Carroll, M. C. Johnson and L. Randall, Dynamical compactification from de Sitter space, JHEP 11 (2009) 094 [0904.3115].

[47] P. Koerber and D. Tsimpis, Supersymmetric sources, integrability and generalized-structure compactifications, JHEP 08 (2007) 082 [0706.1244].

[48] T. Wrase and M. Zagermann, On classical de Sitter vacua in string theory, Fortschr. Phys. 58 (2010) 906-910 [1003.0029].

[49] J. P. Conlon, Brane-Antibrane Backreaction in Axion Monodromy Inflation, 1110.6454 .

[50] S. R. Green, E. J. Martinec, C. Quigley and S. Sethi, Constraints on String Cosmology, 1110.0545, * Temporary entry*. 